Yu. Higuchi and T. Shirai

Nagoya Math. J.

Vol. 161 (2001), 127-154

\title{
WEAK BLOCH PROPERTY FOR DISCRETE MAGNETIC SCHRÖDINGER OPERATORS
}

\author{
YUSUKE HIGUCHI ${ }^{1}$ AND TOMOYUKI SHIRAI ${ }^{2}$
}

\begin{abstract}
For a magnetic Schrödinger operator on a graph, which is a generalization of classical Harper operator, we study some spectral properties: the Bloch property and the behaviour of the bottom of the spectrum with respect to magnetic fields. We also show some examples which have interesting properties.
\end{abstract}

\section{$\S$ 0. Introduction}

The spectral analysis of a discrete Laplacian, regarded as a discrete analogue of the Laplace-Beltrami operator on a Riemannian manifold, has been investigated by many authors (cf. [1], [3], [4], [6]). One of the main topics is to study the relationship between the spectra of discrete Laplacians and the geometries of graphs from many viewpoints. Many researchers have explored the spectra of discrete magnetic Laplacians, each of which is a purturbed Laplacian on a graph by the magnetic field also. For instance, E. Lieb and M. Loss [11] characterized the ground state (in other words, the bottom of the spectrum) of the discrete magnetic Laplacian called $a$ hopping matrix for a finite bipartite planar graph, and T. Sunada [14] gave some criteria for the spectrum which consists of a union of finite number of closed intervals using a twisted group $C^{*}$-algebra for a discrete magnetic Laplacian with a weak invariance under a group action on an infinite graph.

Our main purpose in this paper is to study the spectral properties of the discrete magnetic Laplacian in terms of a growth function for a graph and the behaviour of the bottom of the spectrum as a function of the magnetic flux.

Received April 8, 1999.

Revised September 8, 1999.

2000 Mathematics Subject Classification: Primary 58G25, Secondary 05C50.

${ }^{1}$ Partially supported by the Ministry of Education, Science, Sports and Culture of Japan under the Grant-in-Aid No. 09304022.

${ }^{2}$ Partially supported by Japan Society for the Promotion of Science, Research Fellowships for Young Scientists. 
Now let us explain our setting and the definition of the magnetic Laplacian. Let $G=(V(G), E(G))$ be a connected, locally finite graph, where $V(G)$ is the set of its vertices and $E(G)$ is the set of its unoriented edges. A graph $G$ may have self-loops and multiple edges. Considering each edge in $E(G)$ to have two orientations, we introduce the set of all oriented edges; we denote it by $A(G)$. For an edge $e \in A(G)$, the origin vertex and the terminal one of $e$ are denoted by $o(e)$ and $t(e)$, respectively. The inverse edge of $e$ is denoted by $\bar{e}$. Let $p: A(G) \rightarrow \mathbf{R}^{+}$be a transition probability such that

$$
\sum_{e \in A_{x}(G)} p(e)=1
$$

where $A_{x}(G)=\{e \in A(G) \mid o(e)=x\}$. We assume that $p$ is reversible, that is, there exists a positive valued function $m: V(G) \rightarrow \mathbf{R}^{+}$such that

$$
m(o(e)) p(e)=m(t(e)) p(\bar{e})\left(=: m_{A}(e)\right)
$$

for every oriented edge $e \in A(G)$. The function $m$ is called a reversible measure for $p$ and it is unique, if it exists, up to a multiple constant. Now we set

$$
\ell^{2}(V(G))=\left\{f:\left.V(G) \rightarrow \mathbf{C}\left|\sum_{x \in V(G)}\right| f(x)\right|^{2} m(x)<\infty\right\},
$$

which is a Hilbert space with the inner product

$$
\langle f, g\rangle_{V}=\sum_{x \in V(G)} f(x) \overline{g(x)} m(x) .
$$

Putting

$$
C_{\mathbf{R}}^{1}(G)=\{\theta: A(G) \rightarrow \mathbf{R} \mid \theta(\bar{e})=-\theta(e)\},
$$

we call an element of $C_{\mathbf{R}}^{1}(G)$ a 1-form on $G$. For a fixed 1-form $\theta$, we define a self-adjoint operator $H_{\theta, G}: \ell^{2}(V(G)) \rightarrow \ell^{2}(V(G))$ by

$$
H_{\theta, G} f(x)=\sum_{e \in A_{x}(G)} p(e) \exp (\sqrt{-1} \theta(e)) f(t(e))-f(x) .
$$

This is called a discrete magnetic Schrödinger operator, or simply, a magnetic Laplacian. Some general properties of $H_{\theta}$ will be given in Section 2. It is easy to find that this is a generalization of the classical Harper operator 
on $\mathbf{Z}^{2}$ (Remark 1.6) known as a typical discrete analogue of Schrödinger operators with uniform magnetic fields in $\mathbf{R}^{2}$ (cf. [5], [9], [11], [14]).

We summarize our results described precisely in Section 1. In Theorem A, we give a criterion for a weak version of the Bloch property of $H_{\theta, G}$ using the boundary area growth of a graph $G$ (Definition 1.1). Here a selfadjoint operator $H$ is said to have the (resp. a weak version of the) Bloch property if the set of $\ell^{\infty}$-eigenvalues of $H$ coincides with (resp. is contained in) that of $\ell^{2}$-spectrum. As a corollary of Theorem $\mathrm{A}$, we give a criterion that the spectrum of $H_{\theta, M}$ is contained in that of $H_{\theta, G}$ (Corollary B) when $G$ is a covering graph of a finite graph $M$. In Proposition $\mathrm{C}$, we give the concrete expression of the hessian of $\lambda_{M}(\theta)$, the bottom of the spectrum of $H_{\theta, M}$, at $\theta=0$ for a finite graph $M$. When $G$ is an infinite graph, it is not easy to show some regularity properties of $\lambda_{G}(\theta)$ in $\theta$. In Proposition D, we show that $\lambda_{G}(\theta)$ is continuous in $\theta$ and that it is Fréchet differentiable at $\theta=0$ in the Banach space of bounded 1-forms. In addition, we show an interesting example so-called the "phase transition phenomena" in Example E. Some other examples illustrating our results are given in Section 5.

\section{$\S 1$. Definitions and results}

We give some definitions for stating our results. We use a decomposition $D_{G}$ of $V(G)$ called a 1-dim decomposition and a growth function for $D_{G}$ called a boundary area growth [6].

Definition 1.1. A decomposition $D_{G}$ is called a 1-dim decomposition if it satisfies the following conditions:

a) Suppose $V(G)=\bigcup_{k=0}^{\infty} V_{k}$ where $V_{k}$ is a non-empty finite set of vertices for every $k$,

b) $V_{k}$ 's are mutually disjoint,

c) For any $e \in A(G), o(e) \in V_{k}$ implies $t(e) \in V_{k-1} \cup V_{k} \cup V_{k+1}$.

For a given 1-dim decomposition $D_{G}$, we set $B_{n}=\bigcup_{k=0}^{n} V_{k}$, which is the $n$-ball with respect to $D_{G}$. Put

$$
\begin{aligned}
& \partial B_{n}=\left\{e \in A(G) \mid o(e) \in V_{n}, t(e) \in V_{n+1}\right\}, \\
& \operatorname{Area}\left(\partial B_{n}\right)=\sum_{e \in \partial B_{n}} m_{A}(e),
\end{aligned}
$$

and define the boundary area growth for $D_{G}$ by

$$
\mu\left(D_{G}\right)=\liminf _{n \rightarrow \infty} \frac{1}{n} \log \operatorname{Area}\left(\partial B_{n}\right) .
$$


Remark 1.2. When we set the volume of a finite graph $M$

$$
\operatorname{Vol}(M)=\sum_{x \in V(M)} m(x)
$$

the volume growth $\tilde{\mu}\left(D_{G}\right)$ for $D_{G}$ is defined by

$$
\tilde{\mu}\left(D_{G}\right)=\liminf _{n \rightarrow \infty} \frac{1}{n} \log \operatorname{Vol}\left(B_{n}\right) .
$$

It is easy to see that $\tilde{\mu}\left(D_{G}\right) \geq 0$ and $\mu\left(D_{G}\right) \leq \tilde{\mu}\left(D_{G}\right)$.

For a real-valued bounded function $q$ on $V(G)$, we define a Schrödinger type operator $L_{\theta, G}$ by

$$
L_{\theta, G} u(x)=-H_{\theta, G} u(x)+q(x) u(x) .
$$

Without confusions, we simply denote $H_{\theta, G}$ and $L_{\theta, G}$ by $H_{\theta}$ and $L_{\theta}$, respectively.

We now show a weak version of the Bloch property in terms of the boundary area growth.

TheOrem A. Suppose that an infinite graph $G$ has a 1-dim decomposition $D_{G}$ whose boundary area growth satisfies $\mu\left(D_{G}\right) \leq 0$. If the equation $L_{\theta} u=\lambda u$ has a non-trivial bounded solution, then $\lambda$ is in the spectrum of $L_{\theta}$.

For a given graph, in order to check the assumption of Theorem A, we often need to take a different type of 1-dim decomposition from the most typical one $V(G)=\bigcup_{k=0}^{\infty} V_{k}$, where $V_{k}=\left\{x \in V(G) \mid \operatorname{dist}\left(x, x_{0}\right)=k\right\}$ for a fixed vertex $x_{0} \in V(G)$. Here $\operatorname{dist}(\cdot, \cdot)$ is the shortest path distance.

ExAmple 1.3. Let $P$ be a half infinite path, that is, $V(P)=\left\{x_{i}\right\}_{i=0}^{\infty}$ and $E(P)=\left\{x_{i} x_{i+1}\right\}_{i=0}^{\infty}$. In addition, let $Y$ be a set of vertices $Y=\bigcup_{i=0}^{\infty} Y_{i}$, where $Y_{0}=\emptyset$ and $Y_{i}=\left\{y_{i, k} \mid k=1,2, \ldots, 2^{i}-1\right\}$ for $i \geq 1$. Now we define a graph $G$ as $V(G)=V(P) \cup Y$ and $E(G)=E(P) \cup\left\{x_{i} y \mid y \in Y_{i}\right\}_{i=0}^{\infty}$, that is, $G$ is a tree such that

$$
\operatorname{deg} x= \begin{cases}2^{i}+1, & \text { if } x=x_{i}, \quad i=1,2,3, \ldots \\ 1, & \text { otherwise }\end{cases}
$$


where $\operatorname{deg} x$ denotes the degree of the vertex $x$, which equals to $\# A_{x}(G)$. Let $p(e)=(\operatorname{deg} o(e))^{-1}$ and $m(x)=\operatorname{deg} x$. Here we take two kinds of 1 $\operatorname{dim}$ decompositions of $G: D_{G}$ is the decomposition by the shortest path distance $\operatorname{dist}\left(\cdot, x_{0}\right)$ as is seen above and $D_{G}^{\prime}$ is another decomposition $V(G)=\bigcup_{i=0}^{\infty} V_{i}^{\prime}$, where $V_{i}^{\prime}=\left\{x_{i}\right\} \cup Y_{i}$. Then it is easy to see $\mu\left(D_{G}^{\prime}\right)=0$ while $\mu\left(D_{G}\right)=\log 2$. (One can also see $\tilde{\mu}\left(D_{G}^{\prime}\right)=\tilde{\mu}\left(D_{G}\right)=\log 2$.)

To demonstrate an application of Theorem A, let us assume that $G$ has a group $\Gamma$ of automorphisms which acts on $G$ freely and is finitely generated, and that the quotient space $M=\Gamma \backslash G$ is finite. $M$ has a natural graph structure and we set $M=(V(M), E(M))$.

Suppose that $m, p, \theta$ and $q$ on $G$ are invariant under the $\Gamma$-action. Then we can take functions on $M$ such that

$$
m=m_{0} \circ \pi, \quad p=p_{0} \circ \pi, \quad \theta=\theta_{0} \circ \pi \quad \text { and } \quad q=q_{0} \circ \pi,
$$

where $\pi: G \rightarrow M$ is the natural projection, and so the operator $L_{\theta, G}=$ $-H_{\theta, G}+q$ on $G$ is just the lift of the operator $L_{\theta, M}=-H_{\theta_{0}, M}+q_{0}$ on $M$ by the map $\pi$.

If $\Gamma$ is a group of subexponential growth, then, for the 1-dim decomposition $D_{G}$ by the shortest path distance, it holds that $\tilde{\mu}\left(D_{G}\right)=0$ (cf. [12]); therefore $\mu\left(D_{G}\right) \leq 0$. It is obvious that the lift $\tilde{f}_{\lambda}$ on $G$ of every eigenfunction $f$ for $\lambda$ on $M$ is a non-trivial bounded solution of the equation $L_{\theta, G} \tilde{f}_{\lambda}=\lambda \tilde{f}_{\lambda}$. Hence, we get the following:

Corollary B. If $\Gamma$ is of subexponential growth, then $\operatorname{Spec}\left(L_{\theta, M}\right) \subset$ $\operatorname{Spec}\left(L_{\theta, G}\right)$.

Denoting the bottom of the spectrum of $L_{\theta, G}$ by $\lambda_{G}(\theta)$, it is obvious that $\lambda_{G}(\theta) \leq \lambda_{M}(\theta)$. This consequence includes a discrete analogue of results in [10], where the Bloch property is discussed for the Schrödinger operators on Riemannian manifolds. See also [8].

When $\Gamma$ is of exponential growth, the conclusion above does not hold in general. We have rather obtained the following result:

Theorem 1.4. ([7]) If $\Gamma$ is amenable, then $\operatorname{Spec}\left(L_{\theta, M}\right) \subset \operatorname{Spec}\left(L_{\theta, G}\right)$.

Remark that there exists an amenable group (especially, solvable group) of exponential growth (for instance, [3], [12]) while any group of subexponential growth is amenable. 
The proof of Theorem A will be given in Section 3 .

In the rest of this section, we restrict ourselves to the case where the potential $q=0$ for simplicity. We show two results on the behaviour of $\lambda_{G}(\theta)$ near $\theta=0$ : one is a result for the bottom of the spectrum for a finite graph $M$ and the other is a result which holds in general setting without assuming the covering structure of $G$.

Proposition C. Let $\theta$ be a harmonic 1-form on $M$, that is, $\sum_{e \in A_{x}(M)} p(e) \theta(e)=0$ for every $x \in V(M)$. Then

$$
\left.\frac{d}{d t} \lambda_{M}(t \theta)\right|_{t=0}=0 \quad \text { and }\left.\quad \frac{d^{2}}{d t^{2}} \lambda_{M}(t \theta)\right|_{t=0}=\frac{2\|\theta\|_{A}^{2}}{\operatorname{Vol}(M)},
$$

where $\operatorname{Vol}(M)=\sum_{x \in V(M)} m(x)$ and $\|\theta\|_{A}^{2}=(1 / 2) \sum_{e \in A(M)}|\theta(e)|^{2} m_{A}(e)$.

For an infinite graph $G$, we do not know whether $\lambda_{G}(t \theta)$ is analytic in $t$ or not in general. The following is the partial result:

Proposition D. For an infinite graph $G, \lambda_{G}(\theta) \geq \lambda_{G}(0)$ for any $\theta$. Moreover, let $\ell_{\infty}^{1}(G, \mathbf{R}) \subset C_{\mathbf{R}}^{1}(G)$ be the Banach space of all bounded 1forms with norm $\|\theta\|_{\infty}=\sup _{e \in A(G)} \theta(e)$. Then the bottom $\lambda_{G}$, as a real valued function on $\ell_{\infty}^{1}(G, \mathbf{R})$, is continuous, and Fréchet differentiable at $\theta=0$ and $\lambda_{G}^{\prime}(0)=0$.

When $G$ is a covering graph of a finite graph $M$ and its transformation group $\Gamma$ is abelian, the analyticity of the bottom $\lambda_{G}(\theta)$ near $\theta=0$ is obtained in $[7]$.

The proofs of Proposition $\mathrm{C}$ and Proposition D will be given in Section 4.

Finally in this section, we give an example of the spectrum of $H_{\theta}$. Other examples will be given in Section 5 . We consider the transition probability $p(e)=(\operatorname{deg} o(e))^{-1}$ of a simple random walk on $G$. Then,

$$
H_{\theta} f(x)=(\operatorname{deg} x)^{-1} \sum_{e \in A_{x}(G)} \exp (\sqrt{-1} \theta(e)) f(t(e))-f(x)
$$

for $f \in \ell^{2}(V(G))$. Let $G$ be the 2-dimensional square lattice. We identify every vertex $x \in V(G)$ with a 2 -dimensional vector $(m, n)$ where $m, n \in \mathbf{Z}$ and every edge $x y \in E(G)$ with an unordered pair of vertices $x$ and $y$ satisfying $|x-y|=1$ in the Euclidean metric. We choose an 
orientation $A_{0}(G)=\{e \in A(G) \mid t(e)-o(e)=(1,0)$ or $(0,1)\}$ and then $A(G)=A_{0}(G) \cup \overline{A_{0}(G)}$, where $\overline{A_{0}(G)}=\left\{\bar{e} \in A(G) \mid e \in A_{0}(G)\right\}$. For our convenience, we set $h(x)=m$ and $v(x)=n$ if $x=(m, n)$.

Example E. Set a 1-form $\theta$ on $G$ as follows: for $e \in A_{0}(G)$,

$$
\theta(e)=\left\{\begin{aligned}
s, & \text { if } t(e)-o(e)=(1,0) \text { and } v(o(e)) \text { is even, } \\
-s, & \text { if } t(e)-o(e)=(1,0) \text { and } v(o(e)) \text { is odd } \\
0, & \text { otherwise }
\end{aligned}\right.
$$

and $\theta(e)=-\theta(\bar{e})$ for $e \in \overline{A_{0}(G)}$, where $s$ is real. Then we have

$$
\operatorname{Spec}\left(-H_{\theta}\right)= \begin{cases}{\left[1-\frac{\sqrt{1+1 / \sin ^{2} s}}{2}, 1+\frac{\sqrt{1+1 / \sin ^{2} s}}{2}\right],} \\ \text { if } 0 \leq|\cos s| \leq \frac{-1+\sqrt{5}}{2}, \\ {\left[\frac{1-|\cos s|}{2}, \frac{3+|\cos s|}{2}\right],} & \text { if } \frac{-1+\sqrt{5}}{2} \leq|\cos s| \leq 1 .\end{cases}
$$

Remark 1.5. For every $s$, the operator $H_{\theta}$ in the above has purely absolutely continuous spectrum.

If a path $c=\left(e_{1}, e_{2}, \ldots, e_{n}\right)$, a sequence of oriented edges with $t\left(e_{i}\right)=$ $o\left(e_{i+1}\right)$ for $i=1, \ldots, n-1$, satisfies $t\left(e_{n}\right)=o\left(e_{1}\right)$, then it is said to be a closed path. For a closed path $c$, we define the magnetic flux of $\theta$ through $c$ by

$$
\int_{c} \theta=\sum_{i=1}^{n} \theta\left(e_{i}\right)
$$

Remark 1.6. In Example E, for $(m, n) \in \mathbf{Z}^{2}$, we set the quadrangle cycle $c_{m, n}$ which is a boundary of a unit 2 -cell in $\mathbf{R}^{2}$ as

$$
c_{m, n}=\left(e_{1}, e_{2}, e_{3}, e_{4}\right),
$$

where $o\left(e_{1}\right)=(m, n), o\left(e_{2}\right)=(m+1, n), o\left(e_{3}\right)=(m+1, n+1)$ and $o\left(e_{4}\right)=(m, n+1)$. Then, the magnetic flux through $c_{m, n}$ is as follows:

$$
\int_{c_{m, n}} \theta=\left\{\begin{aligned}
2 s, & \text { if } n \text { is even } \\
-2 s, & \text { if } n \text { is odd }
\end{aligned}\right.
$$


Hence this example corresponds to a "2-periodic magnetic field" case but a uniform magnetic field case. On the other hand, if we choose a 1-form $\theta$ as follows: for $e \in A_{0}(G)$ such that $o(e)=(m, n)$,

$$
\theta(e)=\left\{\begin{aligned}
-n s, & \text { if } t(e)-o(e)=(1,0), \\
0, & \text { otherwise }
\end{aligned}\right.
$$

Then we have

$$
\int_{c_{m, n}} \theta=s
$$

for every $c_{m, n}$, which implies that the corresponding operator is the same as the classical Harper operator. This operator has a complicated spectral structure called Hofstadter's butterfly (for instance, [2], [5], [9]).

Remark 1.7. In Example E, set $\lambda(s)=\inf \operatorname{Spec}\left(-H_{\theta}\right), \alpha=(-1+$ $\sqrt{5}) / 2$ and $s_{c}=\arccos \alpha$. It is obvious that $\lambda(s)$ is real analytic in $s$ if $0<s<s_{c}$ or $s_{c}<s<\pi / 2$. On the other hand, the following hold:

(1) the first derivative for $s, \lambda^{\prime}\left(s_{c}\right)$, exists and $\lambda^{\prime}\left(s_{c}\right)=\sqrt{\alpha} / 2$,

(2) the second derivative, $\lambda^{\prime \prime}\left(s_{c}\right)$, does not exist.

The phenomena at $s=s_{c}$ may be considered as a kind of "second order phase transition".

\section{$\S$ 2. The magnetic Laplacians}

In this section, we state some elementary properties of a magnetic Laplacian and its spectrum.

Let $G$ be a connected, locally finite graph. For a given 1 -form $\theta \in$ $C_{\mathbf{R}}^{1}(G)$, put

$$
\begin{aligned}
& C^{0}(G)=\{f: V(G) \rightarrow \mathbf{C}\}, \\
& C^{1}(G)=\{\phi: A(G) \rightarrow \mathbf{C} \mid \phi(\bar{e})=-\exp (-\sqrt{-1} \theta(e)) \phi(e) \text { for } e \in A(G)\},
\end{aligned}
$$

and define the linear operators $d_{\theta}: C^{0}(G) \rightarrow C^{1}(G)$ and $\delta_{\theta}: C^{1}(G) \rightarrow$ $C^{0}(G)$ as follows:

$$
\begin{aligned}
& \left(d_{\theta} f\right)(e)=\exp (\sqrt{-1} \theta(e)) f(t(e))-f(o(e)) \quad \text { for } f \in C^{0}(G) \\
& \left(\delta_{\theta} \phi\right)(x)=-\sum_{e \in A_{x}(G)} p(e) \phi(e) \text { for } \phi \in C^{1}(G)
\end{aligned}
$$


We easily find that the magnetic Laplacian $H_{\theta, G}$ defined by $(0.1)$ can be expressed as $-\delta_{\theta} d_{\theta}$ :

$$
\begin{aligned}
H_{\theta, G} f(x) & =\left(-\delta_{\theta} d_{\theta} f\right)(x) \\
& =\sum_{e \in A_{x}(G)} p(e)\{\exp (\sqrt{-1} \theta(e)) f(t(e))-f(x)\} \\
& =\sum_{e \in A_{x}(G)} p(e) \exp (\sqrt{-1} \theta(e)) f(t(e))-f(x) .
\end{aligned}
$$

We set

$$
\ell^{2}(V(G))=\left\{f \in C^{0}(G) \mid\langle f, f\rangle_{V}<\infty\right\},
$$

where the inner product $\left\langle f_{1}, f_{2}\right\rangle_{V}=\sum_{x \in V(G)} f_{1}(x) \overline{f_{2}(x)} m(x)$, and set

$$
\ell^{2}(A(G))=\left\{\phi \in C^{1}(G) \mid\langle\phi, \phi\rangle_{A}<\infty\right\},
$$

where the inner product $\left\langle\phi_{1}, \phi_{2}\right\rangle_{A}=(1 / 2) \sum_{e \in A(G)} \phi_{1}(e) \overline{\phi_{2}(e)} m_{A}(e)$. Here we denote by $m_{A}(e)$ the quantity $m(o(e)) p(e)$. Remark that $m_{A}$ is symmetric, that is, $m_{A}(e)=m_{A}(\bar{e})$. Then it is easy to check $\left\langle d_{\theta} f, \phi\right\rangle_{A}=\left\langle f, \delta_{\theta} \phi\right\rangle_{V}$ and $\left\langle d_{\theta} f, d_{\theta} f\right\rangle_{A} \leq 2\langle f, f\rangle_{V}$ for any $f \in \ell^{2}(V(G))$ and $\phi \in \ell^{2}(A(G))$. Therefore, the magnetic Laplacian $H_{\theta, G}$ is a bounded self-adjoint operator on $\ell^{2}(V(G))$ and the spectrum of $-H_{\theta, G}, \operatorname{Spec}\left(-H_{\theta, G}\right)$, is a closed subset in $[0,2]$.

If $\theta(e)=0$ for any $e \in A(G), H_{\theta, G}$ is just a discrete Laplacian $\Delta$ (cf. [1], $[3],[4],[6]):$

$$
\Delta f(x)=\sum_{e \in A_{x}(G)} p(e) f(t(e))-f(x) .
$$

Lemma 2.1. For a given 1-form $\theta \in C_{\mathbf{R}}^{1}(G)$, put $\theta^{\prime}(e)=-\theta(e)$ for every $e \in A(G)$. Then $\operatorname{Spec}\left(-H_{\theta}\right)=\operatorname{Spec}\left(-H_{\theta^{\prime}}\right)$.

Proof. It is obvious by setting a unitary map $U: \ell^{2}(V(G)) \rightarrow \ell^{2}(V(G))$ as $U(f)=\bar{f}$.

LEMma 2.2. Choose and fix any orientation $A_{0}(G)$ on $E(G)$ such that $A(G)=A_{0}(G) \cup \overline{A_{0}(G)}$, where $\overline{A_{0}(G)}=\left\{\bar{e} \in A(G) \mid e \in A_{0}(G)\right\}$. For a given 1-form $\theta$, set a 1-form $\theta^{\prime}$ as follows: $\theta^{\prime}(e)=\pi-\theta(e)$ for every $e \in A_{0}(G)$ and $\theta^{\prime}(e)=-\theta^{\prime}(\bar{e})$ for every $e \in \overline{A_{0}(G)}$. Then $\operatorname{Spec}\left(-H_{\theta}\right)$ and $\operatorname{Spec}\left(-H_{\theta^{\prime}}\right)$ are symmetric with respect to 1 , that is, $\operatorname{Spec}\left(I+H_{\theta}\right)=$ $-\operatorname{Spec}\left(I+H_{\theta^{\prime}}\right)$. 
Proof. Since $\exp \left(\sqrt{-1} \theta^{\prime}(e)\right)=-\exp (-\sqrt{-1} \theta(e))$, it holds that $(I+$ $\left.H_{\theta^{\prime}}\right) f(x)=-\overline{\left(I+H_{\theta}\right) \bar{f}}(x)$. From Lemma 2.1, we get $\operatorname{Spec}\left(I+H_{\theta}\right)=$ $-\operatorname{Spec}\left(I+H_{\theta^{\prime}}\right)$.

A graph $G$ is called bipartite if there exists a partition $V(G)=V\left(G_{0}\right) \cup$ $V\left(G_{1}\right)$ such that $V\left(G_{0}\right)$ and $V\left(G_{1}\right)$ are disjoint sets of vertices and either $o(e) \in V\left(G_{0}\right)$ and $t(e) \in V\left(G_{1}\right)$ or $o(e) \in V\left(G_{1}\right)$ and $t(e) \in V\left(G_{0}\right)$ occurs for any $e \in A(G)$. Equivalently, a graph $G$ is bipartite if and only if every closed path of $G$ has even length. For a bipartite graph, the next lemma is basic.

LEMma 2.3. Suppose that a graph $G$ is bipartite. Then $\operatorname{Spec}\left(-H_{\theta}\right)$ is symmetric with respect to 1 , that is, $\lambda \in \operatorname{Spec}\left(I+H_{\theta}\right)$ if and only if $-\lambda \in$ $\operatorname{Spec}\left(I+H_{\theta}\right)$.

Proof. For bipartiteness of $G$, we can take a partition $V(G)=V\left(G_{0}\right) \amalg$ $V\left(G_{1}\right)$. Define the unitary map $U: \ell^{2}(V(G)) \rightarrow \ell^{2}(V(G))$ by

$$
(U f)(x)=\left\{\begin{aligned}
f(x), & \text { if } x \in V\left(G_{0}\right), \\
-f(x), & \text { if } x \in V\left(G_{1}\right) .
\end{aligned}\right.
$$

Then we obtain $\left(U^{-1}\left(I+H_{\theta}\right) U\right) f(x)=-\left(I+H_{\theta}\right) f(x)$.

The next statement is essentially the same as Lemma 2.1 in [11], which states that the magnetic fluxes (cf. (1.8)) determine the spectrum.

LEMmA 2.4. Let $\theta_{1}, \theta_{2} \in C_{\mathbf{R}}^{1}(G)$ be 1-forms on $G$. If the magnetic flux of $\theta_{1}$ equals to that of $\theta_{2}$ in modulo $2 \pi$ for every closed path of $G$, then $\operatorname{Spec}\left(-H_{\theta_{1}}\right)=\operatorname{Spec}\left(-H_{\theta_{2}}\right)$.

Proof. Choose and fix any vertex $x_{0} \in V(G)$. We define the function $\psi(x)$ on $V(G)$ as follows: for any vertex $x \in V(G)$, take a path $p=\left(e_{1}, e_{2}, \ldots e_{n}\right)$ such that $o\left(e_{1}\right)=x_{0}$ and $t\left(e_{n}\right)=x$, and set

$$
\psi(x)=\exp \left(\sqrt{-1} \int_{x_{0}}^{x}\left(\theta_{1}-\theta_{2}\right)\right) .
$$

It is easy to show that $\psi(x)$ does not depend on the choice of a path from $x_{0}$ to $x$. Indeed, take $p_{1}$ and $p_{2}$ from $x_{0}$ to $x$ and consider the closed path 
$c=p_{1} \overline{p_{2}}$, where $\overline{p_{2}}$ is the inverse directed path of $p_{2}$. It follows from the assumption that

$$
\int_{p_{1}}\left(\theta_{1}-\theta_{2}\right)-\int_{p_{2}}\left(\theta_{1}-\theta_{2}\right)=\int_{c}\left(\theta_{1}-\theta_{2}\right) \in 2 \pi \mathbf{Z} .
$$

Therefore we have

$$
\exp \left(\sqrt{-1} \int_{p_{1}}\left(\theta_{1}-\theta_{2}\right)\right)=\exp \left(\sqrt{-1} \int_{p_{2}}\left(\theta_{1}-\theta_{2}\right)\right)
$$

which implies the independence of the choice of a path.

Define a unitary map $U: \ell^{2}(V(G)) \rightarrow \ell^{2}(V(G))$ by $U f(x)=\psi(x) f(x)$. Then we have

$$
\begin{aligned}
H_{\theta_{1}} f(x) & =\sum_{e \in A_{x}(G)} p(e) \exp \left(\sqrt{-1} \theta_{1}(e)\right) f(t(e))-f(x) \\
& =\sum_{e \in A_{x}(G)} p(e)(\psi(x))^{-1} \exp \left(\sqrt{-1} \theta_{2}(e)\right) \psi(t(e)) f(t(e))-f(x) \\
& =(\psi(x))^{-1}\left(\sum_{e \in A_{x}(G)} p(e) \exp \left(\sqrt{-1} \theta_{2}(e)\right) \psi(t(e)) f(t(e))-\psi(x) f(x)\right) \\
& =\left(U^{-1} H_{\theta_{2}} U f\right)(x),
\end{aligned}
$$

hence we get $\operatorname{Spec}\left(-H_{\theta_{1}}\right)=\operatorname{Spec}\left(-H_{\theta_{2}}\right)$.

Remark 2.5. If $G$ is a tree, the magnetic flux is zero for any closed path and for any 1 -form $\theta$. Therefore it holds that $\operatorname{Spec}\left(-H_{\theta}\right)=\operatorname{Spec}(-\Delta)$ for any 1 -form $\theta$.

\section{$\S 3$. Weak Bloch property: Theorem A}

In this section, we give the proof of Theorem A stated in Section 1 and we observe that the weak Bloch property for the discrete magnetic Schrödinger operator $L_{\theta}$ holds under the condition of the boundary area growth for a 1-dim decomposition.

Proof of Theorem A. From the assumption, we can take a 1-dim decomposition $D_{G}$ whose boundary area growth satisfies $\mu\left(D_{G}\right) \leq 0$ and fix it. Let $u$ be a non-trivial bounded solution of the equation $L_{\theta} u=\lambda u$. For the $n$-ball $B_{n}$ with respect to $D_{G}$, put

$$
j(n)=\sum_{e \in \partial B_{n}} m_{A}(e)\left(|u(o(e))|^{2}+|u(t(e))|^{2}\right)
$$


and

$$
J(n)=\sum_{x \in B_{n}} m(x)|u(x)|^{2} .
$$

Recall that we denote the quantity $m(o(e)) p(e)$ by $m_{A}(e)$. Since $u$ is bounded and $J(n) \geq C>0$ for any sufficiently large $n$, we have

$$
\liminf _{n \rightarrow \infty} \frac{1}{n} \log j(n) \leq 0 \leq \liminf _{n \rightarrow \infty} \frac{1}{n} \log J(n) .
$$

Now we define the function $\chi_{n}$ by

$$
\chi_{n}(x)= \begin{cases}1, & \text { if } x \in B_{n}, \\ 0, & \text { if } x \in V(G) \backslash B_{n},\end{cases}
$$

and put

$$
f_{n}=u \cdot \chi_{n}
$$

Moreover, for $B_{n} \subset V(G)$, put

$$
B_{n}^{0}=\left\{x \in B_{n} \mid t(e) \in B_{n} \text { for all } e \in A_{x}(G)\right\},
$$

$\partial_{0} B_{n}=\left\{x \in B_{n} \mid\right.$ there exists an $e \in A_{x}(G)$ such that $\left.t(e) \in V(G) \backslash B_{n}\right\}$, $\partial_{1} B_{n}=\left\{x \in V(G) \backslash B_{n} \mid\right.$ there exists an $e \in A_{x}(G)$ such that $\left.t(e) \in B_{n}\right\}$.

Then we have the following lemma:

LEMMA 3.1.

$$
\left(L_{\theta}-\lambda\right) f_{n}(x)= \begin{cases}0, \quad \text { if } x \in B_{n}^{0} \text { or } x \in V(G) \backslash\left\{B_{n} \cup \partial_{1} B_{n}\right\}, \\ \sum_{\substack{e \in A_{x}(G) \\ t(e) \in \partial_{1} B_{n}}} p(e) \exp (\sqrt{-1} \theta(e)) u(t(e)), \quad \text { if } x \in \partial_{0} B_{n}, \\ \sum_{\substack{e \in A_{x}(G) \\ t(e) \in \partial_{0} B_{n}}}-p(e) \exp (\sqrt{-1} \theta(e)) u(t(e)), & \text { if } x \in \partial_{1} B_{n} .\end{cases}
$$

Proof of Lemma 3.1. 1) If $x \in B_{n}^{0}$, then we have $\left(L_{\theta}-\lambda\right) f_{n}(x)=\left(L_{\theta}-\right.$ $\lambda) u(x)=0$. 2) If $x \in V(G) \backslash\left\{B_{n} \cup \partial_{1} B_{n}\right\}$, then we have $f_{n}(x)=0$ and 
$f_{n}(t(e))=0$ for any $e \in A_{x}(G)$. So, $\left.\left(L_{\theta}-\lambda\right) f_{n}(x)=0.3\right)$ If $x \in \partial_{0} B_{n}$, then we get

$$
\begin{aligned}
& \left(L_{\theta}-\lambda\right) f_{n}(x) \\
& =(1+q(x)-\lambda) f_{n}(x)-\sum_{e \in A_{x}(G)} p(e) \exp (\sqrt{-1} \theta(e)) f_{n}(t(e)) \\
& =(1+q(x)-\lambda) u(x)-\sum_{\substack{e \in A_{x}(G) \\
t(e) \in B_{n}}} p(e) \exp (\sqrt{-1} \theta(e)) f_{n}(t(e))
\end{aligned}
$$

since $f_{n}(t(e))=0$ for $t(e) \in \partial_{1} B_{n}$. Moreover, since $u$ is a solution to $L_{\theta} u=$ $\lambda u$, we get

$$
\begin{aligned}
0 & =\left(L_{\theta}-\lambda\right) u(x) \\
& =(1+q(x)-\lambda) u(x)-\sum_{e \in A_{x}(G)} p(e) \exp (\sqrt{-1} \theta(e)) u(t(e)) .
\end{aligned}
$$

Hence we obtain

$$
\left(L_{\theta}-\lambda\right) f_{n}(x)=\sum_{\substack{e \in A_{x}(G) \\ t(e) \in \partial_{1} B_{n}}} p(e) \exp (\sqrt{-1} \theta(e)) u(t(e))
$$

4) If $x \in \partial_{1} B_{n}$, then $f_{n}(x)=0$. Therefore we get

$$
\begin{aligned}
\left(L_{\theta}-\lambda\right) f_{n}(x) & =-\sum_{e \in A_{x}(G)} p(e) \exp (\sqrt{-1} \theta(e)) f_{n}(t(e)) \\
& =-\sum_{\substack{e \in A_{x}(G) \\
t(e) \in \partial_{0} B_{n}}} p(e) \exp (\sqrt{-1} \theta(e)) u(t(e)) .
\end{aligned}
$$

This completes the proof of Lemma 3.1.

From Lemma 3.1, we obtain

$$
\begin{aligned}
& \left\|\left(L_{\theta}-\lambda\right) f_{n}\right\|^{2} \\
& =\sum_{x \in \partial_{0} B_{n}} m(x)\left|\sum_{\substack{e \in A_{x}(G) \\
t(e) \in \partial_{1} B_{n}}} p(e) \exp (\sqrt{-1} \theta(e)) u(t(e))\right|^{2} \\
& \quad+\sum_{x \in \partial_{1} B_{n}} m(x)\left|-\sum_{\substack{e \in A_{x}(G) \\
t(e) \in \partial_{0} B_{n}}} p(e) \exp (\sqrt{-1} \theta(e)) u(t(e))\right|^{2} .
\end{aligned}
$$


By the Cauchy-Schwarz inequality, we estimate the first term of the right hand side of (3.11) as follows:

$$
\begin{aligned}
& \sum_{x \in \partial_{0} B_{n}} m(x)\left|\sum_{\substack{e \in A_{x}(G) \\
t(e) \in \partial_{1} B_{n}}} p(e) \exp (\sqrt{-1} \theta(e)) u(t(e))\right|^{2} \\
& \leq \sum_{x \in \partial_{0} B_{n}} m(x)\left(\sum_{\substack{e \in A_{x}(G) \\
t(e) \in \partial_{1} B_{n}}} p(e)\right)\left(\sum_{\substack{e \in A_{x}(G) \\
t(e) \in \partial_{1} B_{n}}} p(e)|u(t(e))|^{2}\right) \\
& \leq \sum_{x \in \partial_{0} B_{n}} \sum_{\substack{e \in A_{x}(G) \\
t(e) \in \partial_{1} B_{n}}} m_{A}(e)|u(t(e))|^{2} .
\end{aligned}
$$

Similarly, we have

$$
\begin{gathered}
\sum_{x \in \partial_{1} B_{n}} m(x)\left|-\sum_{\substack{e \in A_{x}(G) \\
t(e) \in \partial_{0} B_{n}}} p(e) \exp (\sqrt{-1} \theta(e)) u(t(e))\right|^{2} \\
\leq \sum_{x \in \partial_{1} B_{n}} \sum_{\substack{e \in A_{x}(G) \\
t(e) \in \partial_{0} B_{n}}} m_{A}(e)|u(t(e))|^{2}
\end{gathered}
$$

Due to (3.11), (3.12) and (3.13), we have

$$
\begin{aligned}
\left\|\left(L_{\theta}-\lambda\right) f_{n}\right\|^{2} \leq & \sum_{x \in \partial_{0} B_{n}} \sum_{\substack{e \in A_{x}(G) \\
t(e) \in \partial_{1} B_{n}}} m_{A}(e)|u(t(e))|^{2} \\
& \quad+\sum_{x \in \partial_{1} B_{n}} \sum_{\substack{e \in A_{x}(G) \\
t(e) \in \partial_{0} B_{n}}} m_{A}(e)|u(t(e))|^{2} \\
\leq & \sum_{e \in \partial B_{n}} m_{A}(e)\left(|u(o(e))|^{2}+|u(t(e))|^{2}\right)=j(n) .
\end{aligned}
$$

For our purpose, it suffices to show that, for any $\epsilon>0$, there exists a nonzero function $f \in \ell^{2}(V(G))$ such that

$$
\left\|\left(L_{\theta}-\lambda\right) f\right\|^{2} \leq \epsilon\|f\|^{2} .
$$

The proof is divided into two cases: 1$) \liminf _{n \rightarrow \infty}(1 / n) \log J(n)>0$ and 2) $\liminf _{n \rightarrow \infty}(1 / n) \log J(n)=0$. 
1) Let us first consider the case that $\liminf _{n \rightarrow \infty}(1 / n) \log J(n)>0$. It follows from this condition that, for any positive number $\delta$ such that $\delta<\liminf _{n \rightarrow \infty}(1 / n) \log J(n)$, there exists a subsequence $\left\{n_{k}\right\}$ satisfying

$$
\frac{1}{n_{k}} \log j\left(n_{k}\right)<\frac{\delta}{2}, \quad \frac{1}{n_{k}} \log J\left(n_{k}\right)>\delta .
$$

Due to (3.16), we get

$$
\frac{j\left(n_{k}\right)}{J\left(n_{k}\right)}<\exp \left(-n_{k} \cdot \delta / 2\right)
$$

Remark that $J(n)=\left\|f_{n}\right\|^{2}$ for every $n$. Therefore, by (3.14) and (3.17),

$$
\frac{\left\|\left(L_{\theta}-\lambda\right) f_{n_{k}}\right\|^{2}}{\left\|f_{n_{k}}\right\|^{2}} \leq \frac{j\left(n_{k}\right)}{J\left(n_{k}\right)}<\exp \left(-n_{k} \cdot \delta / 2\right) .
$$

Hence we can select $n_{k}$ so that $\exp \left(-n_{k} \cdot \delta / 2\right) \leq \epsilon$, which implies $\lambda$ is in the spectrum of $L_{\theta}$.

2) Consider the case that $\liminf _{n \rightarrow \infty}(1 / n) \log J(n)=0$. Under this condition, we first show that, for any positive number $\delta$, there exists $n$ such that

$$
J(n+1) \leq \exp (2 \delta) \cdot J(n-1) .
$$

Suppose that there would exist $\delta>0$ such that

$$
J(n+1)>\exp (2 \delta) \cdot J(n-1)
$$

for all $n$. Then we have

$$
\begin{aligned}
J(n+1) & >\exp (2 \delta) \cdot J(n-1)>\exp (4 \delta) \cdot J(n-3)>\cdots \\
& >\exp \left(2\left[\left(n+1-n_{0}\right) / 2\right] \delta\right) \cdot J\left(n+1-2\left[\left(n+1-n_{0}\right) / 2\right]\right) \\
& >\exp \left(\left(n-n_{0}-1\right) \delta\right) \cdot J\left(n_{0}\right)
\end{aligned}
$$

for any $n>n_{0}$, where $n_{0}$ is an integer such that $J\left(n_{0}\right)>0$, and we consequently obtain that

$$
\liminf _{n \rightarrow \infty} \frac{1}{n} \log J(n) \geq \delta>0 .
$$

This is a contradiction, and hence we have (3.19). 
Due to (3.19), we get

$$
\begin{aligned}
j(n) & =\sum_{e \in \partial B_{n}} m_{A}(e)\left(|u(o(e))|^{2}+|u(t(e))|^{2}\right) \\
& \leq \sum_{x \in \partial_{0} B_{n} \cup \partial_{1} B_{n}} m(x)|u(x)|^{2} \leq J(n+1)-J(n-1) \\
& \leq(\exp (2 \delta)-1) \cdot J(n-1) \leq(\exp (2 \delta)-1) \cdot J(n) .
\end{aligned}
$$

Therefore, by (3.14) and (3.21), we have

$$
\left\|\left(L_{\theta}-\lambda\right) f_{n}\right\|^{2} \leq j(n) \leq(\exp (2 \delta)-1)\left\|f_{n}\right\|^{2}
$$

Hence, choosing $\delta$ so that $(\exp (2 \delta)-1) \leq \epsilon$, we obtain the desired estimate (3.15).

The proof of Theorem A is now completed.

\section{$\S 4$. Some remarks on the bottom of the spectrum: Propositions $\mathrm{C}$ and D}

In this section, we give the proofs of Proposition $\mathrm{C}$ and Proposition D in Section 1.

Define $d: C^{0}(M) \rightarrow C^{1}(M)$ and $\delta: C^{1}(M) \rightarrow C^{0}(M)$ by

$$
d f(e)=f(t(e))-f(o(e)) \text { and } \quad \delta \omega(x)=-\sum_{e \in A_{x}(M)} p(e) \omega(e)
$$

respectively. As in Section 2, one can immediately find that $d=d_{\theta}$ and $\delta=\delta_{\theta}$ for the 1 -form $\theta=0$, and that $d$ and $\delta$ are mutually adjoint operators with respect to $\langle\cdot, \cdot\rangle_{V}$ and $\langle\cdot, \cdot\rangle_{A}$. We now set

$$
\begin{aligned}
& C_{\mathbf{R}}^{0}(M)=\{f: V(M) \rightarrow \mathbf{R}\} \\
& C_{\mathbf{R}}^{1}(M)=\{\omega: A(M) \rightarrow \mathbf{R} \mid \omega(\bar{e})=-\omega(e)\}
\end{aligned}
$$

It is obvious that $d f \in C_{\mathbf{R}}^{1}(M)$ for any $f \in C_{\mathbf{R}}^{0}(M)$ and $\delta \omega \in C_{\mathbf{R}}^{0}(M)$ for any $\omega \in C_{\mathbf{R}}^{1}(M)$. We call $\omega \in C_{\mathbf{R}}^{1}(M)$ a harmonic 1-form if $\delta \omega=0$.

For our convenience, we define the perturbed transition operator $P(\theta)$ : $\ell^{2}(V(M)) \rightarrow \ell^{2}(V(M))$ by

$$
P(\theta) f(x)=\sum_{e \in A_{x}(M)} p(e) \exp (\sqrt{-1} \theta(e)) f(t(e)) .
$$


Of course, $-H_{\theta, M}=I-P(\theta)$.

Now, we fix $\theta \in C_{\mathbf{R}}^{1}(M)$ and consider the operator $P(t \theta)$; we write $P_{t}$ for it. We denote by $\mu(t)$ the largest eigenvalue of $P_{t}$. Remark that $\mu(0)=1$, $\mu(0)$ is simple and its eigenfunction is a constant function. It is well-known in the analytic perturbation theory (cf. [13]) that for sufficiently small $|t|<\epsilon$, $\mu(t)$ is simple and analytic in $t$.

The next proposition implies Proposition C.

Proposition 4.1. For $\mu(t)$ as above, we have

$$
\begin{aligned}
& \mu^{\prime}(0)=0 \\
& \mu^{\prime \prime}(0)=\frac{-2}{\operatorname{Vol}(M)}\left\{\left\langle\Delta_{0}^{-1} \delta \theta, \delta \theta\right\rangle_{V}+\|\theta\|_{A}^{2}\right\},
\end{aligned}
$$

where $\Delta_{0}$ is the restriction of $\Delta(=P(0)-I)$ to the orthogonal complement of the space of constant functions in $\ell^{2}(V(M))$. In particular, when $\theta \in$ $C_{\mathbf{R}}^{1}(M)$ is a harmonic 1-form, we have

$$
\mu^{\prime \prime}(0)=-\frac{2}{\operatorname{Vol}(M)}\|\theta\|_{A}^{2} .
$$

Proof. Let $f_{t}$ be the eigenfunction for the eigenvalue $\mu(t)$ satisfying $f_{0} \equiv 1$. We differentiate the both sides of the equation

$$
\left\langle P_{t} f_{t}, 1\right\rangle_{V}=\mu(t)\left\langle f_{t}, 1\right\rangle_{V}
$$

First we compute

$$
\begin{aligned}
& \left\langle P_{t} f_{t}, 1\right\rangle_{V}=\sum_{x \in V(M)} m(x) \sum_{e \in A_{x}(M)} p(e) \exp (\sqrt{-1} t \theta(e)) f_{t}(t(e)) \\
& =\sum_{x \in V(M)} m(x) \sum_{e \in A_{x}(M)} p(e)\left(1+\sqrt{-1} t \theta(e)-\left(t^{2} / 2\right) \theta(e)^{2}\right) f_{t}(t(e)) \\
& \quad+O\left(t^{3}\right) \\
& =\left\langle P(0) f_{t}, 1\right\rangle_{V}+\sqrt{-1} t \sum_{e \in A(M)} m(o(e)) p(e) \theta(e)\left(d f_{t}(e)+f_{t}(o(e))\right) \\
& \quad-\frac{t^{2}}{2} \sum_{e \in A(M)} m_{A}(e) \theta(e)^{2} f_{t}(t(e))+O\left(t^{3}\right) \\
& =\left\langle f_{t}, 1\right\rangle_{V}+\sqrt{-1} t\left(2\left\langle d f_{t}, \theta\right\rangle_{A}-\left\langle f_{t}, \delta \theta\right\rangle_{V}\right)
\end{aligned}
$$




$$
\begin{aligned}
& -\frac{t^{2}}{2} \sum_{e \in A(M)} m_{A}(e) \theta(e)^{2} f_{t}(t(e))+O\left(t^{3}\right) \\
=\left\langle f_{t}, 1\right\rangle_{V}+\sqrt{-1} t\left\langle d f_{t}, \theta\right\rangle_{A} & \\
& -\frac{t^{2}}{2} \sum_{e \in A(M)} m_{A}(e) \theta(e)^{2} f_{t}(t(e))+O\left(t^{3}\right) .
\end{aligned}
$$

Then we have

$$
\left.\frac{d}{d t}\left\langle P_{t} f_{t}, 1\right\rangle_{V}\right|_{t=0}=\left\langle f_{0}^{\prime}, 1\right\rangle_{V}
$$

and

$$
\left.\frac{d}{d t} \mu(t)\left\langle f_{t}, 1\right\rangle_{V}\right|_{t=0}=\mu^{\prime}(0)\left\langle f_{0}, 1\right\rangle_{V}+\mu(0)\left\langle f_{0}^{\prime}, 1\right\rangle_{V}
$$

Then we have $\mu^{\prime}(0)=0$.

Similarly, we have

$$
\begin{aligned}
\left.\frac{d^{2}}{d t^{2}}\left\langle P_{t} f_{t}, 1\right\rangle_{V}\right|_{t=0} & =\left\langle f_{0}^{\prime \prime}, 1\right\rangle_{V}+2 \sqrt{-1}\left\langle d f_{0}^{\prime}, \theta\right\rangle_{A}-\sum_{e \in A(M)} m_{A}(e) \theta(e)^{2} f_{0}(t(e)) \\
& =\left\langle f_{0}^{\prime \prime}, 1\right\rangle_{V}+2 \sqrt{-1}\left\langle d f_{0}^{\prime}, \theta\right\rangle_{A}-2\|\theta\|_{A}^{2}
\end{aligned}
$$

On the other hand, we have

$$
\begin{aligned}
\left.\frac{d^{2}}{d t^{2}} \mu(t)\left\langle f_{t}, 1\right\rangle_{V}\right|_{t=0} & =\mu^{\prime \prime}(0)\left\langle f_{0}, 1\right\rangle_{V}+2 \mu^{\prime}(0)\left\langle f_{0}^{\prime}, 1\right\rangle_{V}+\mu(0)\left\langle f_{0}^{\prime \prime}, 1\right\rangle_{V} \\
& =\mu^{\prime \prime}(0)\langle 1,1\rangle_{V}+\left\langle f_{0}^{\prime \prime}, 1\right\rangle_{V} .
\end{aligned}
$$

Then we obtain that

$$
\mu^{\prime \prime}(0)=\frac{2}{\operatorname{Vol}(M)}\left\{\sqrt{-1}\left\langle d f_{0}^{\prime}, \theta\right\rangle_{A}-\|\theta\|_{A}^{2}\right\} .
$$

Now we remark the equation

$$
\Delta f_{0}^{\prime}=\sqrt{-1} \delta \theta
$$

which is obtained by differentiating the both sides of the equality $P_{t} f_{t}=$ $\mu(t) f_{t}$ at $t=0$. Since $\langle\delta \theta, 1\rangle_{V}=\langle\theta, d 1\rangle_{A}=0$, or equivalently, $\delta \theta$ is orthogonal to the constant functions, the equation (4.10) can be solved. Then, setting $\Delta_{0}=\left.\Delta\right|_{\ell^{2}(V(M)) \ominus \mathbf{C}}$, we have

$$
f_{0}^{\prime}=\sqrt{-1} \Delta_{0}^{-1} \delta \theta
$$

Combining (4.9) and (4.11), we complete the proof. 
Next we give the proof of Proposition D.

Proof of Proposition D. Assume that $\|f\|=1$. For any 1 -forms $\theta_{1}, \theta_{2} \in$ $\ell_{\infty}^{1}(G, \mathbf{R}) \subset C_{\mathbf{R}}^{1}(G)$, it holds that

$$
\begin{aligned}
\lambda_{G}\left(\theta_{1}\right) & \leq\left\langle-H_{\theta_{2}} f, f\right\rangle_{V}+\left\langle-\left(H_{\theta_{1}}-H_{\theta_{2}}\right) f, f\right\rangle_{V} \\
& \leq\left\langle-H_{\theta_{2}} f, f\right\rangle_{V}+\left\|H_{\theta_{1}}-H_{\theta_{2}}\right\| \\
& \leq\left\langle-H_{\theta_{2}} f, f\right\rangle_{V}+\left\|\theta_{1}-\theta_{2}\right\|_{\infty} .
\end{aligned}
$$

Then we have

$$
\left|\lambda_{G}\left(\theta_{1}\right)-\lambda_{G}\left(\theta_{2}\right)\right| \leq\left\|\theta_{1}-\theta_{2}\right\|_{\infty}
$$

and so $\lambda_{G}(\theta)$ is continuous in $\theta$.

Since

$$
\begin{aligned}
\left\langle-H_{\theta} f, f\right\rangle_{V} & =\left\langle d_{\theta} f, d_{\theta} f\right\rangle_{A} \\
& =\frac{1}{2} \sum_{e \in A(G)} m_{A}(e)|\exp (\sqrt{-1} t \theta(e)) f(t(e))-f(o(e))|^{2} \\
& \geq\left.\frac{1}{2} \sum_{e \in A(G)} m_{A}(e)|| f(t(e))|-| f(o(e))\right|^{2} \\
& =\left\langle-H_{0}|f|,|f|\right\rangle_{V} \geq \lambda_{G}(0)
\end{aligned}
$$

we have

$$
\lambda_{G}(\theta) \geq \lambda_{G}(0)
$$

As $\theta \rightarrow 0$ in $\ell_{\infty}^{1}(G, \mathbf{R})$, we obtain

$$
\begin{aligned}
\lambda_{G}(\theta) \leq & \left\langle-H_{0} f, f\right\rangle_{V}+\left\langle-\left(H_{\theta}-H_{0}\right) f, f\right\rangle_{V} \\
= & \left\langle-H_{0} f, f\right\rangle_{V}+\sum_{e \in A(G)} m_{A}(e) \theta(e) \operatorname{Im}(f(o(e)) \overline{f(t(e))}) \\
& \quad+O\left(\|\theta\|_{\infty}^{2}\right),
\end{aligned}
$$

where $O\left(\|\theta\|_{\infty}^{2}\right)$ is independent of $f$ when $\|f\|=1$. Note that

$$
\lambda_{G}(0)=\inf _{\|f\|=1}\left\langle-H_{0} f, f\right\rangle_{V}=\inf _{\substack{\|f\|=1 \\ f: \text { real-valued }}}\left\langle-H_{0} f, f\right\rangle_{V} .
$$

Then, by taking the infimum over real-valued functions in (4.14), we obtain

$$
\lambda_{G}(\theta) \leq \lambda_{G}(0)+O\left(\|\theta\|_{\infty}^{2}\right) .
$$


Consequently, by (4.13) and (4.15), we get

$$
\lambda_{G}^{\prime}(0)=0
$$

This completes the proof.

\section{$\S 5$. Examples}

In this section, we consider three infinite graphs as $G$; the square lattice, the triangular one and the hexagonal one. Then, we give some examples of the spectrum of $H_{\theta, G}$,

$$
H_{\theta, G} f(x)=(\operatorname{deg} x)^{-1} \sum_{e \in A_{x}(G)} \exp (\sqrt{-1} \theta(e)) f(t(e))-f(x)
$$

where $\operatorname{deg} x=\# A_{x}(G)$ denotes the degree of the vertex $x$ in $G$.

For each $G$, we also consider a finite graph $M$ which is a quotient graph $\Gamma \backslash G$, where $\Gamma$ is an abelian group of automorphisms acting on $G$, and an infinite tree $T$ which is the universal covering of $M$ (therefore, of $G$ ). For a 1 -form $\theta$ which is $\Gamma$-invariant on $G$, let $\tilde{\theta}$ be the lift of $\theta$ to $T$ and $\theta_{0}$ the natural projection of $\theta$ onto $M$ as in (1.6). We observe the spectra of $H_{\tilde{\theta}, T}$, $H_{\theta, G}$ and $H_{\theta_{0}, M}$.

Let $T_{d}$ be the $d$-regular tree. It is easy to see that $T=T_{4}, T_{6}$ and $T_{3}$ if $G$ is the square, the triangular and the hexagonal lattice, respectively. Furthermore, it follows from Remark 2.5 that $H_{\tilde{\theta}, T}$ and $H_{0, T}\left(=\Delta_{T}\right)$ are unitarily equivalent for any 1-form $\theta$ and it is well-known (cf. [3]) that $\Delta_{T_{d}}$ has purely absolutely continuous spectrum, which is given by

$$
\operatorname{Spec}\left(-\Delta_{T_{d}}\right)=[1-2 \sqrt{d-1} / d, 1+2 \sqrt{d-1} / d]
$$

Remark 5.1. For any infinite graph $G$ in our examples, every spectrum set of $-H_{\theta, G}$ is purely absolutely continuous.

\subsection{The square lattice}

Let $G$ be the 2-dimensional square lattice. Recall that $h(x)=m$ and $v(x)=n$ if $x=(m, n)$. Now we take a finite quotient graph $M=\Gamma \backslash G$, where $\Gamma=3 \mathbf{Z} \oplus 2 \mathbf{Z}$. (See Figure 5.1.) We identify $V(M)$ with the set $\left\{(m, n) \in \mathbf{Z}^{2} \mid m \in\{0,1,2\}\right.$ and $\left.n \in\{0,1\}\right\}$ and set $A_{0}(M)=\Gamma \backslash A_{0}(G)$. 


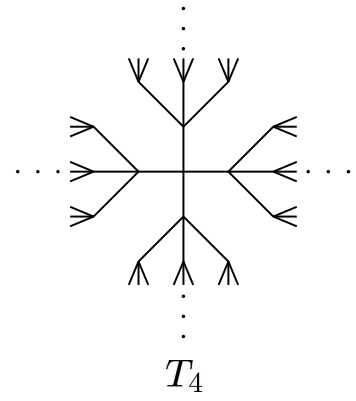

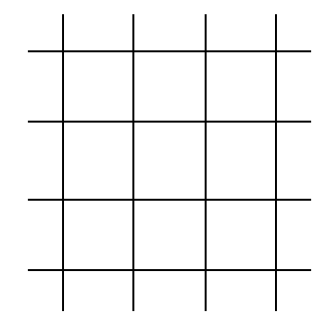

$G$

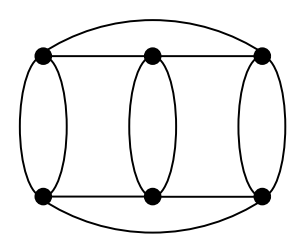

$M$

Figure 5.1: $T_{4}, G=\mathbf{Z}^{2}$ and $M$.

ExAmPLE 5.2. (parallel flow) Set a 1-form $\theta$ on $A(G)$ as follows: for $e \in A_{0}(G)$,

$$
\theta(e)= \begin{cases}s, & \text { if } t(e)-o(e)=(1,0) \\ 0, & \text { otherwise }\end{cases}
$$

and $\theta(e)=-\theta(\bar{e})$ for $e \in \overline{A_{0}(G)}$. Then it is obvious that

$$
\operatorname{Spec}\left(-H_{\theta, G}\right)=[0,2]
$$

for all $s$ since $\int_{c_{m, n}} \theta=0$ for every $m, n$. See Remark 1.6 and Lemma 2.4. We set a 1 -form $\theta_{0}$ as a natural projection of $\theta$ onto $A(M)$ : for $e \in A_{0}(M)$,

$$
\theta_{0}(e)= \begin{cases}s, & \text { if } t(e)-o(e) \equiv(1,0) \bmod 3 \mathbf{Z} \oplus 2 \mathbf{Z} \\ 0, & \text { otherwise }\end{cases}
$$

Then we have

$$
\operatorname{Spec}\left(-H_{\theta_{0}, M}\right)=\left\{\frac{1-\cos s}{2}, \frac{3-\cos s}{2}, \frac{1+\cos \left(s \pm \frac{\pi}{3}\right)}{2}, \frac{3+\cos \left(s \pm \frac{\pi}{3}\right)}{2}\right\} .
$$

Set $\lambda_{G}(s)=\inf \operatorname{Spec}\left(-H_{\theta, G}\right)$ and $\lambda_{M}(s)=\inf \operatorname{Spec}\left(-H_{\theta_{0}, M}\right)$. Remark that $\lambda_{G}(s)=\lambda_{M}(s)$ when $s=0$ and $2 \pi / 3$.

ExAmPLE 5.3. (alternate flow) Set a 1-form $\theta$ on $A(G)$ as follows: for $e \in A_{0}(G)$,

$$
\theta(e)=\left\{\begin{aligned}
s, & \text { if } v(o(e)) \text { is even and } t(e)-o(e)=(1,0) \\
-s, & \text { if } v(o(e)) \text { is odd and } t(e)-o(e)=(1,0) \\
0, & \text { otherwise, }
\end{aligned}\right.
$$


and $\theta(e)=-\theta(\bar{e})$ for $e \in \overline{A_{0}(G)}$. Then, for $0 \leq s \leq \pi / 2$, we have

$$
\operatorname{Spec}\left(-H_{\theta, G}\right)=\left\{\begin{array}{l}
{\left[\frac{1-\cos s}{2}, \frac{3+\cos s}{2}\right], \quad \text { if } 0 \leq s \leq s_{c},} \\
{\left[1-\frac{\sqrt{1+1 / \sin ^{2} s}}{2}, 1+\frac{\sqrt{1+1 / \sin ^{2} s}}{2}\right]} \\
\text { if } s_{c} \leq s \leq \pi / 2,
\end{array}\right.
$$

where $s_{c}=\arccos ((-1+\sqrt{5}) / 2)$. We set a 1 -form $\theta_{0}$ as a natural projection of $\theta$ onto $A(M)$ : for $e \in A_{0}(M)$,

$$
\theta_{0}(e)=\left\{\begin{aligned}
s, & \text { if } v(o(e))=0 \text { and } t(e)-o(e) \equiv(1,0) \bmod 3 \mathbf{Z} \oplus 2 \mathbf{Z} \\
-s, & \text { if } v(o(e))=1 \text { and } t(e)-o(e) \equiv(1,0) \bmod 3 \mathbf{Z} \oplus 2 \mathbf{Z} \\
0, & \text { otherwise. }
\end{aligned}\right.
$$

Then we have

$$
\begin{aligned}
& \operatorname{Spec}\left(-H_{\theta_{0}, M}\right) \\
& =\left\{\frac{1-\cos s}{2}, \frac{3-\cos s}{2}, \frac{4+\cos s \pm \sqrt{7-\cos ^{2} s}}{4}, \frac{4+\cos s \pm \sqrt{7-\cos ^{2} s}}{4}\right\} .
\end{aligned}
$$

As is seen in Section $1, \lambda_{G}(s)$ is in $C^{1}$ but the second derivative does not exist at $s=s_{c}$. Remark that $\lambda_{G}(s)=\lambda_{M}(s)$ when $0 \leq s \leq s_{c}$ and $s=\arccos ((\sqrt{5}-\sqrt{13}) / 4)$.

In Figure 5.2, we give the graphs of the behaviour of $\lambda .(s)$ : the left figure is for Example 5.2 and the right one for Example 5.3.
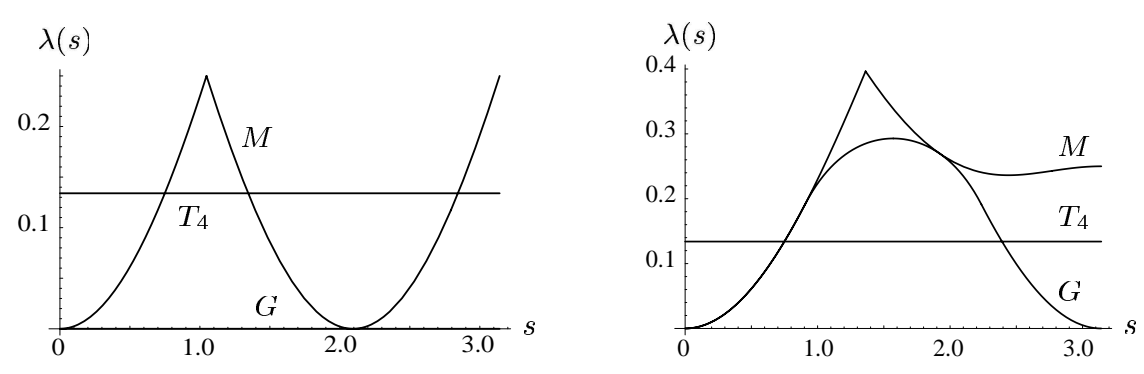

Figure 5.2: the graphs of $\lambda .(s)$. 


\subsection{The triangular lattice}

Let $G$ be the triangular lattice. We identify $x \in V(G)$ with a 2dimensional vector $(m, n)$ where $m, n \in \mathbf{Z}$, and set an ordered pair of vertices $x$ and $y$ by $x y \in A(G)$ if $x$ and $y$ satisfy one of the following: $y-x=(0, \pm 1),( \pm 1,0)$ or $\pm(1,1)$. Choose $A_{0}(G)=\{e \in A(G)$ $t(e)-o(e)=(1,0),(1,1)$ or $(0,1)\}$. We take a finite quotient graph $M=$ $\Gamma \backslash G$, where $\Gamma=3 \mathbf{Z} \oplus 2 \mathbf{Z}$. (See Figure 5.3.) We identify $V(M)$ with the set $\left\{(m, n) \in \mathbf{Z}^{2} \mid m \in\{0,1,2\}\right.$ and $\left.n \in\{0,1\}\right\}$ and set $A_{0}(M)=\Gamma \backslash A_{0}(G)$.

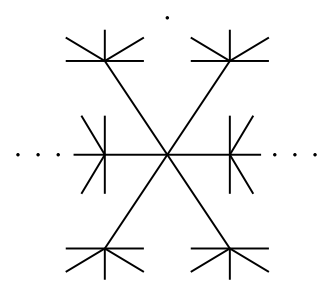

$T_{6}$

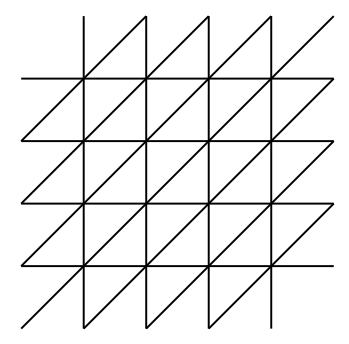

G

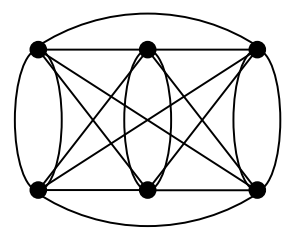

$M$

Figure 5.3: $T_{6}, G$ and $M$.

EXAmple 5.4. (parallel flow) Set a 1-form $\theta$ on $A(G)$ as follows: for $e \in A_{0}(G)$,

$$
\theta(e)= \begin{cases}s, & \text { if } t(e)-o(e)=(1,0), \\ 0, & \text { otherwise }\end{cases}
$$

and $\theta(e)=-\theta(\bar{e})$ for $e \in \overline{A_{0}(G)}$. Then, for $0 \leq s \leq \pi$, we have

$$
\operatorname{Spec}\left(-H_{\theta, G}\right)=[1-\cos (s / 3), 1-\cos (s / 3+2 \pi / 3)] .
$$

We set a 1 -form $\theta_{0}$ as a natural projection of $\theta$ onto $A(M)$ : for $e \in A_{0}(M)$,

$$
\theta_{0}(e)= \begin{cases}s, & \text { if } t(e)-o(e) \equiv(1,0) \bmod 3 \mathbf{Z} \oplus 2 \mathbf{Z}, \\ 0, & \text { otherwise }\end{cases}
$$

Then we have

$$
\operatorname{Spec}\left(-H_{\theta_{0}, M}\right)=\left\{\frac{1-\cos s}{3}, \frac{5-\cos s}{3}, \frac{5+2 \cos \left(s \pm \frac{\pi}{3}\right)}{6}, \frac{7+\cos \left(s \pm \frac{\pi}{3}\right)}{6}\right\} .
$$


ExAmPLE 5.5. (alternate flow) Set a 1-form $\theta$ on $A(G)$ as follows: for $e \in A_{0}(G)$,

$$
\theta(e)= \begin{cases}s, & \text { if } v(o(e)) \text { is even and } t(e)-o(e)=(1,0), \\ -s, & \text { if } v(o(e)) \text { is odd and } t(e)-o(e)=(1,0) \\ 0, & \text { otherwise }\end{cases}
$$

and $\theta(e)=-\theta(\bar{e})$ for $e \in \overline{A_{0}(G)}$. Then, for $0 \leq s \leq \pi$, we have

$$
\operatorname{Spec}\left(-H_{\theta, G}\right)= \begin{cases}{\left[\frac{1-\cos s}{3}, \frac{5+4 \cos s}{3+3 \cos s}\right],} & \text { if } 0 \leq s \leq \pi / 2, \\ {\left[\frac{1-2 \cos s}{3-3 \cos s}, \frac{5-\cos s}{3}\right],} & \text { if } \pi / 2 \leq s \leq \pi .\end{cases}
$$

We set a 1 -form $\theta_{0}$ as a natural projection of $\theta$ onto $A(M)$ : for $e \in A_{0}(M)$,

$$
\theta_{0}(e)= \begin{cases}s, & \text { if } v(o(e))=0 \text { and } t(e)-o(e) \equiv(1,0) \bmod 3 \mathbf{Z} \oplus 2 \mathbf{Z}, \\ -s, & \text { if } v(o(e))=1 \text { and } t(e)-o(e) \equiv(1,0) \bmod 3 \mathbf{Z} \oplus 2 \mathbf{Z} \\ 0, & \text { otherwise. }\end{cases}
$$

Then we have

$$
\begin{aligned}
\operatorname{Spec}\left(-H_{\theta_{0}, M}\right)=\left\{\frac{1-\cos s}{3}, \frac{5-\cos s}{3}, \frac{1+\left(\cos s \pm \sqrt{4-3 \cos ^{2} s}\right)}{6},\right. \\
\left.\frac{1+\left(\cos s \pm \sqrt{4-3 \cos ^{2} s}\right)}{6}\right\} .
\end{aligned}
$$

It is obvious that $\lambda_{G}(s)$ is real analytic in $s$ if $0<s<\pi / 2$ or $\pi / 2<$ $s<\pi$ and that $\lambda_{G}^{\prime}(\pi / 2)=1 / 3$ and $\lambda_{G}^{\prime \prime}(\pi / 2)$ does not exist.

In Figure 5.4, the left figure is for Example 5.4 and the right one for Example 5.5.

\subsection{The hexagonal lattice}

Let $G$ be the hexagonal lattice. We identify $x \in V(G)$ with a 2dimensional vector $(m, n)$ where $m, n \in \mathbf{Z}$ and set an ordered pair of vertices $x$ and $y$ by $x y \in A(G)$ if $x$ and $y$ satisfy one of the following: 1) $y-x=$ $( \pm 1,0)$ or $(0,1)$ if $h(x)+v(x)$ is even; 2) $y-x=( \pm 1,0)$ or $(0,-1)$ if $h(x)+$ $v(x)$ is odd. Choose $A_{0}(G)=\{e \in A(G) \mid t(e)-o(e)=(1,0)$ or $(0,1)\}$. We take a finite quotient graph $M=\Gamma \backslash G$, where $\Gamma=4 \mathbf{Z} \oplus 2 \mathbf{Z}$. (See Figure 5.5.) We identify $V(M)$ with the set $\left\{(m, n) \in \mathbf{Z}^{2} \mid m \in\{0,1,2,3\}\right.$ and $\left.n \in\{0,1\}\right\}$ and set $A_{0}(M)=\Gamma \backslash A_{0}(G)$. 

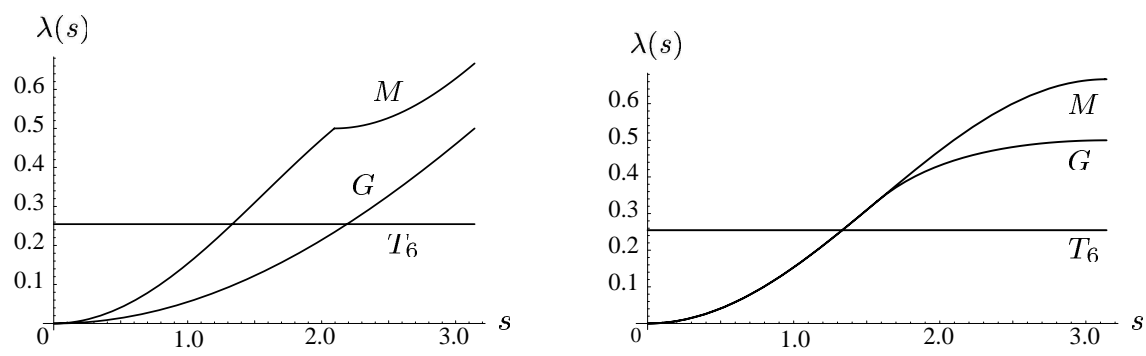

Figure 5.4: the graphs of $\lambda .(s)$.

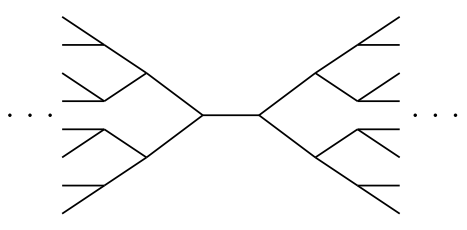

$T_{3}$

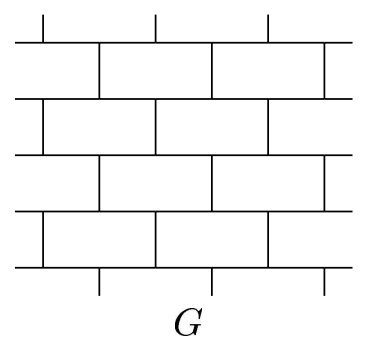

$G$

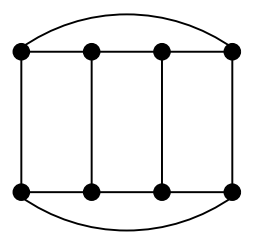

$M$

Figure 5.5: $T_{3}, G$ and $M$.

For the hexagonal lattice $G$, it is easy to see that $H_{\theta, G}$ and $H_{0, G}$ are unitarily equivalent in the "parallel flow" case as in Example 5.2. Thus we observe the spectra in the "alternate flow" case only.

EXAMPLE 5.6. (alternate flow) Set a 1-form $\theta$ on $A(G)$ as follows: for $e \in A_{0}(G)$,

$$
\theta(e)=\left\{\begin{aligned}
s, & \text { if } v(o(e)) \text { is even and } t(e)-o(e)=(1,0), \\
-s, & \text { if } v(o(e)) \text { is odd and } t(e)-o(e)=(1,0), \\
0, & \text { otherwise, }
\end{aligned}\right.
$$

and $\theta(e)=-\theta(\bar{e})$ for $e \in \overline{A_{0}(G)}$. Then, for $0 \leq s \leq \pi / 4$, we have

$$
\operatorname{Spec}\left(-H_{\theta, G}\right)=\left\{\begin{array}{l}
{\left[\frac{2-2 \cos s}{3}, \frac{4+2 \cos s}{3}\right], \quad \text { if } 0 \leq s \leq s_{c},} \\
{\left[1-\frac{\sqrt{4+1 / \sin ^{2} s}}{3}, 1+\frac{\sqrt{4+1 / \sin ^{2} s}}{3}\right],} \\
\text { if } s_{c} \leq s \leq \pi / 4,
\end{array}\right.
$$


where $s_{c}=\arccos \alpha$ and $\alpha=(-1+\sqrt{17}) / 4$. We set a 1 -form $\theta_{0}$ as a natural projection of $\theta$ onto $A(M)$ : for $e \in A_{0}(M)$,

$$
\theta_{0}(e)=\left\{\begin{aligned}
s, & \text { if } v(o(e))=0 \text { and } t(e)-o(e) \equiv(1,0) \bmod 4 \mathbf{Z} \oplus 2 \mathbf{Z}, \\
-s, & \text { if } v(o(e))=1 \text { and } t(e)-o(e) \equiv(1,0) \bmod 4 \mathbf{Z} \oplus 2 \mathbf{Z}, \\
0, & \text { otherwise. }
\end{aligned}\right.
$$

Then we have

$$
\begin{aligned}
& \operatorname{Spec}\left(-H_{\theta_{0}, M}\right) \\
& \quad=\left\{\frac{2 \pm 2 \cos s}{3}, \frac{4 \pm 2 \cos s}{3}, 1 \pm \frac{\sqrt{5-4 \cos ^{2} s}}{3}, 1 \pm \frac{\sqrt{5-4 \cos ^{2} s}}{3}\right\} .
\end{aligned}
$$

It is easy to see that $\operatorname{Spec}\left(-H_{\theta, G}\right)$ is symmetric with respect to $s=\pi / 4$. It is obvious that $\lambda_{G}(s)$ is real analytic in $s$ if $0<s<s_{c}$ or $s_{c}<s<\pi / 4$ and that $\lambda_{G}^{\prime}\left(s_{c}\right)=\sqrt{2 \alpha} / 3$ and $\lambda_{G}^{\prime \prime}\left(s_{c}\right)$ does not exist.

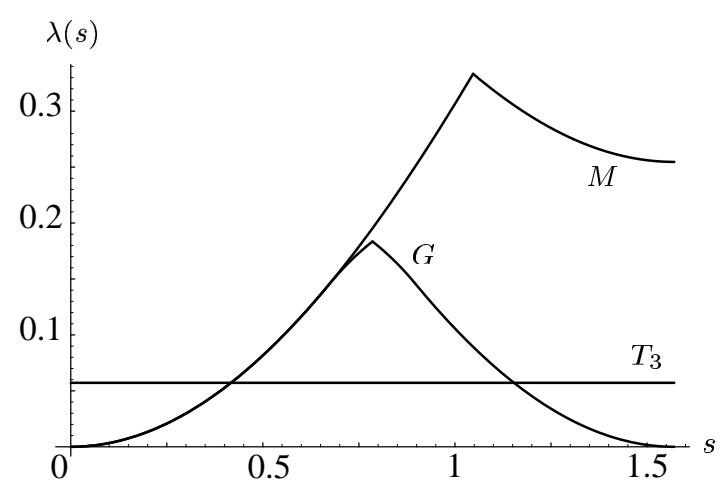

Figure 5.6: the graphs of $\lambda .(s)$.

Acknowledgements. The authors wish to thank Professors Hitoshi Hirata, Toshikazu Sunada and Yoichiro Takahashi for their valuable comments. The authors also had many helpful suggestions by the anonymous referee and Professor Norihiko Kamiura.

\section{REFERENCES}

[1] R. Brooks, Combinatorial problems in spectral geometry, in the Proc. of Taniguchi Symp. "Curvature and topology of Riemannian manifolds" 1985, Lecture Note in Math., 1201 (1986), 14-32. 
[2] M. D. Choi, G. Elliott and N. Yui, Gauss polynomials and the rotation algebra, Invent. Math., 99 (1990), 225-246.

[3] J. Dodziuk and L. Karp, Spectral and function theory for combinatorial Laplacians, Contemp. Math., 73 (1988), 25-40.

[4] J. Dodziuk and W. S. Kendall, Combinatorial Laplacians and isoperimetric inequality, in "From local times to global geometry, control and physics" (K. D. Elworthy, ed.), Pitman Research Notes in Mathematics Series, 150 (1986), 68-74.

[5] P. G. Harper, Single band motion of conduction electrons in a uniform magnetic field, Proc. Phys. Soc. London, A68 (1955), 874-878.

[6] Yu. Higuchi, Boundary area growth and the spectrum of discrete Laplacian, preprint.

[7] Yu. Higuchi and T. Shirai, The spectrum of magnetic Schrödinger operators on a graph with periodic structure, J. Funct. Anal., 169 (1999), 456-480.

[8] — A remark on the spectrum of magnetic Laplacian on a graph, Yokohama Math. J., 47 Special Issue (1999), 129-141.

[9] D. R. Hofstadter, Energy levels and wave functions of Bloch electrons in rational and irrational magnetic fields, Phys. Rev., B14 (1976), 2239-2249.

[10] T. Kobayashi, K. Ono and T. Sunada, Periodic Schrödinger operators on a manifold, Forum Math., 1 (1989), 69-79.

[11] E. Lieb and M. Loss, Fluxes, Laplacians and Kasteleyn's theorem, Duke Math. J., 71 (1993), 337-363.

[12] J. Milnor, A note on curvature and fundamental group, J. Differ. Geom., 2 (1968), $1-7$.

[13] M. C. Reed and B. Simon, Methods of Modern Mathematical Physics Vol. IV, Academic Press, New York, 1978.

[14] T. Sunada, A discrete analogue of periodic magnetic Schrödinger operators, in "Geometry of the spectrum" (P. Parry, R. Brooks, C. Gordon, eds.), Contemporary Mathematics, 173 (1994), 283-299.

Yusuke Higuchi

Mathematics Laboratories

College of Arts and Sciences

Showa University

4562 Kamiyoshida

Fujiyoshida

Yamanashi, 403-0005

Japan

higuchi@cas.showa-u.ac.jp 
Tomoyuki Shirai

Department of Mathematics

Tokyo Institute of Technology

Oh-okayama

Meguro-ku

Tokyo, 152-8551

Japan

shirai@math.titech.ac.jp 